\title{
A Group Theoretic Interpretation of the Last Part of de Branges' Proof of the Bieberbach Conjecture
}

TOM H. KOORNWINDER

Centre for Mathematics and Computer Science, P.O. Box 4079, 1009 AB Amsterdam. The Netherlands

Communicated by Ch. Pommerenke

\begin{abstract}
A more conceptual and less computational proof is given for the last part of de Branges' proof of the Bieberbach conjecture, i.e. where the special functions enter and the AskeyGasper inequality is applied. General solutions of de Branges' system of differential equations are brought in 1-1 correspondence first with Fourier-sine series and next with spherical function expansions on the sphere $S^{3}$. Restriction of spherical functions on $S^{5}$ to $S^{3}$ and positive definiteness then finish the proof.
\end{abstract}

AMS (MOS): 33A75, 30C50, 33A45, 43A35, 43A90

\section{INTRODUCTION}

Several people asked me about a possible group theoretic interpretation for the last part of de Branges' proof of the Bieberbach conjecture [3], i.e. for the part where the special functions enter and the Askey-Gasper [1] inequality is applied. A partial answer was given in Askey and Gasper [2, $\S 2]$. They reduce the question of the positivity of the hypergeometric functions

$$
{ }_{3} F_{2}\left(n-r, r+n+2, n+\frac{1}{2} ; 2 n+1, n+\frac{3}{2} ; s^{-1}\right),
$$

$s \geqslant 1, n, r \in \mathbb{Z}, 1 \leqslant n \leqslant r$, to proving that for each $n, r$ the expansion

$$
C_{r-n}^{n+1}(x)=\sum_{l=n}^{r} a_{l} C_{l-n}^{(1 / 2)+n}(x)
$$


holds with nonnegative coefficients $a_{k}$. Here $C_{m}^{\lambda}(x)$ is a Gegenbauer polynomial. This nonnegativity follows without computation by observing that (1.2) can be interpreted as restricting a (zonal) spherical function on the sphere $S^{2 n+3}=\mathrm{SO}(2 n+4) / \mathrm{SO}(2 n+3)$ to $S^{2 n+2}$ and then expanding it in terms of the spherical functions on $S^{2 n+2}$. Since the zonal positive definite functions on a sphere are precisely the functions having spherical function expansions with nonnegative coefficients and restrictions of positive definite functions on a sphere to a lower dimensional sphere are again positive definite, the nonnegativity of the $a_{k}$ 's follows. If next, in (1.2), $x$ is replaced by $1-(1-x) s^{-1}$ and both sides are multiplied by $\left(1-x^{2}\right)^{n-(1 / 2)}$ and integrated with respect to $x$ from -1 to 1 then, at the left, the ${ }_{3} F_{2}$ in (1.1) appears while, at each term at the right, $\mathrm{a}_{3} F_{2}$ arises which, by Clausen's identity, can be written as

$$
\text { const }\left(C_{l-n}^{n+(1 / 2)}\left(\left(1-s^{-1}\right)^{1 / 2}\right)\right)^{2}
$$

with positive constant.

Two elements remain unsatisfactory about this Askey-Gasper approach. First, de Branges [4] and Koornwinder [8] show that (1.2) is not really needed for all $n$ but only for $n=1$, so it is a matter of restriction of spherical functions on $S^{5}$ to $S^{4}$. However, this reduction is performed by a trick which needs deeper explanation. Second, one would like to have a better understanding why ${ }_{3} F_{2}$ functions arise which can be written as squares.

In the present paper I will explain both things. The key observation is that general solutions of de Branges' system of differential equations are obtained as Fourier-sine coefficients of functions of argument transformed under the action of a one-parameter semigroup. This can be rewritten in group theoretic form, where rotation groups $\mathrm{SO}(6), \mathrm{SO}(5)$, $\mathrm{SO}(4), \mathrm{SO}(3)$ are involved and then an extension of the positive definiteness argument we mentioned yields the result. I conclude the paper with a similar, but not group theoretic characterization of the solutions of de Branges' more general system of differential equations in [4].

It is tempting to extend the group theoretic interpretation presented here to the earlier parts of the proof of the Bieberbach conjecture. For instance, do univalent analytic functions on the disk conceptually live on a low dimensional sphere and can Loewner's differential equation be interpreted in group language? However, if anything is possible in this spirit then it must be tied up with the logarithmic case. It follows from 
[4] that $v$ th powers of univalent functions are connected with "spherical functions" on "spheres" of fractional dimension $2 v+4$, the case $v=0$ being the logarithmic case.

Many mathematicians have checked de Branges' proof of the Bieberbach conjecture, but did not have the courage to do the (not too tedious) computations leading to the Askey-Gasper inequality for themselves. The proof presented here may serve as a less computational and more conceptual alternative. The remaining computations only involve trigonometric identities.

For convenience of the reader without knowledge about positive definite and spherical functions on compact groups, Section 3 is inserted with the relevant material.

\section{AN INTEGRAL TRANSFORM YIELDING THE GENERAL SOLUTION OF DE BRANGES' SYSTEM OF DIFFERENTIAL EQUATIONS}

In [3] de Branges considered the system of differential equations

$$
\sigma_{n}(t)+n^{-1} t \sigma_{n}^{\prime}(t)=\sigma_{n+1}(t)-(n+1)^{-1} t \sigma_{n+1}^{\prime}(t),
$$

$t \geqslant 1, n=1,2, \ldots$. Call a solution $\left\{\sigma_{n}\right\}$ admissible if $\sigma_{1}$ is not identically zero, $\sigma_{n}$ is identically zero for $n$ sufficiently large and $\sigma_{n}^{\prime}(t) \leqslant 0$ for all $t \geqslant 1, n=1,2, \ldots$. In [3, Theorem 2] de Branges states that any admissible solution of (2.1) yields a Milin type inequality for the logarithmic power series coefficients of a univalent analytic function on the unit disk which sends 0 to 0 . The Milin inequality itself follows by showing that the unique solution of (2.1) with initial values

$$
\sigma_{n}(1)=\max (r+1-n, 0), \quad n=1,2, \ldots
$$

is admissible. For this solution we have that $-s^{n+1} \sigma_{n}^{\prime}(s)$ equals a positive factor times expression (1.1), cf. [3, proof of Theorem 3], but this will not be needed here. By (2.2) and (2.1):

$$
-\sigma_{n}^{\prime}(1)= \begin{cases}n, & n=r, r-2, r-4, \ldots, \\ 0, & \text { otherwise. }\end{cases}
$$

Note that, if $\left\{\sigma_{n}\right\}$ is a solution of (2.1) then other solutions are given by the functions $t \mapsto \sigma_{n}(s t)(s \geqslant 1)$ and $t \mapsto t \sigma_{n}^{\prime}(t)$. 
TheOREM 2.1 Let $P \in C^{1}([-1,1])$ and define functions $\sigma_{n}=\sigma_{n}[P]$ $(n=1,2, \ldots)$ on $[1, \infty)$ by

$$
\sigma_{n}(t):=2 \pi^{-1} t^{-1} \int_{0}^{\pi} P\left(1-t^{-1}+t^{-1} \cos \theta\right) \sin (n \theta) \sin \theta d \theta .
$$

Then the functions $\sigma_{n}$ solve (2.1) with initial values

$$
\sigma_{n}(1)=2 \pi^{-1} \int_{0}^{\pi} P(\cos \theta) \sin (n \theta) \sin \theta d \theta .
$$

Proof Let $\sigma_{n}(t)$ be defined by (2.4). Straightforward integration by parts yields

$$
\begin{aligned}
& \sigma_{n}(t)+ n^{-1} t \sigma_{n}^{\prime}(t)-\sigma_{n+1}(t)+(n+1)^{-1} \sigma_{n+1}^{\prime}(t) \\
&=2 \pi^{-1} t^{-1} \int_{0}^{\pi} P\left(1-t^{-1}+t^{-1} \cos \theta\right) \\
& \times\left[\frac{n-1}{n} \sin (n \theta) \sin \theta+\frac{1}{n} \frac{\partial}{\partial \theta}(\sin (n \theta)(1-\cos \theta))\right. \\
&\left.\quad-\frac{n+2}{n+1} \sin (n+1) \theta \sin \theta+\frac{1}{n+1} \frac{\partial}{\partial \theta}(\sin (n+1) \theta(1-\cos \theta))\right] d \theta .
\end{aligned}
$$

It follows from elementary trigonometric identities that the expression in square brackets vanishes.

Observe that

$\left(\sigma_{n}[P]\right)(s t)=\left(\sigma_{n}\left[P_{s}\right]\right)(t), \quad$ where $\quad P_{s}(x):=s^{-1} P\left(1-s^{-1}+s^{-1} x\right), \quad s \geqslant$ and

$$
-t\left(\sigma_{n}[P]\right)^{\prime}(t)=\left(\sigma_{n}[Q]\right)(t), \quad \text { where } \quad Q(x):=\frac{d}{d x}((x-1) P(x))
$$

If a solution $\left\{\sigma_{n}\right\}$ of (2.1) is identically zero for $n$ large then, by (2.5), $\sigma_{n}=\sigma_{n}[P]$ with $P$ given by the terminating series

$$
P(\cos \theta)=\sum_{n=1}^{\infty} \sigma_{n}(1) \frac{\sin (n \theta)}{\sin \theta},
$$

while, in view of $(2.6),-t \sigma_{n}^{\prime}(t)=\left(\sigma_{n}[Q]\right)(t)$ with $Q$ given by the terminating series

$$
Q(\cos \theta)=\sum_{n=1}^{\infty}\left(-\sigma_{n}^{\prime}(1)\right) \frac{\sin (n \theta)}{\sin \theta} .
$$


Obviously

$$
U_{n-1}(\cos \theta):=\frac{\sin (n \theta)}{\sin \theta}
$$

defines a polynomial $U_{n-1}$ of degree $n-1$ which satisfies the orthogonality relations

$$
\frac{2}{\pi} \int_{-1}^{1} U_{k}(x) U_{l}(x)\left(1-x^{2}\right)^{1 / 2} d x=\delta_{k, l}
$$

(Chebyshev polynomials of the second kind). So (2.7) (or (2.8)) is terminating iff $P$ (or $Q$ ) is a polynomial. If $Q$ is a polynomial then, by (2.6), it determines a unique polynomial $P$ given by

$$
P(x)=(1-x)^{-1} \int_{x}^{1} Q(y) d y,
$$

and (2.8) will be valid with $\sigma_{n}:=\sigma_{n}[P]$. We can now conclude:

Proposition 2.2 Formula (2.8) establishes a 1-1 correspondence between admissible solutions $\left\{\sigma_{n}\right\}$ of (2.1) and nonzero polynomials $Q$ such that $\left(\sigma_{n}[Q]\right)(t) \geqslant 0$ for all $t \geqslant 1, n=1,2, \ldots$.

If $\left\{\sigma_{n}\right\}$ is the solution of (2.1) determined by (2.2) then (2.3) and (2.8) yield

$$
\begin{aligned}
Q(\cos \theta) & =\sum_{k=0}^{[(1 / 2)(r-1)]}(r-2 k) \frac{\sin (r-2 k) \theta}{\sin \theta} \\
& =\sum_{k=0}^{[(1 / 2)(r-1)]} U_{r-1-2 k}(\cos \theta) U_{r-1-2 k}(1) .
\end{aligned}
$$

Hence $Q$ is a polynomial of degree $r-1, Q(-x)=(-1)^{r-1} Q(x)$ and, by (2.10) and (2.12), we have for any polynomial $p$ of degree $\leqslant r$

$$
\int_{-1}^{1} Q(x) p(x)\left(1-x^{2}\right)^{1 / 2} d x=p(1) \text { or } 0
$$

according to whether $Q$ and $p$ have the same or opposite parity, respectively. It follows that $Q$ is an orthogonal polynomial of degree $r-1$ on the interval $(-1,1)$ with respect to the weight function $\left(1-x^{2}\right)^{3 / 2}$, normalized by

$$
Q(1)=\sum_{k=0}^{[(1 / 2)(r-1)]}(r-2 k)^{2}=\frac{1}{6} r(r+1)(r+2) .
$$


Generally, Gegenbauer polynomials $C_{n}^{\lambda}$ are orthogonal polynomials of degree $n$ on the interval $(-1,1)$ with respect to the weight function $\left(1-x^{2}\right)^{i-(1 / 2)}$, normalized by

$$
C_{n}^{i}(1)=\frac{(2 \lambda)_{n}}{n !},
$$

where $(a)_{k}:=a(a+1) \cdots(a+k-1)$. Hence $Q$ given by (2.12) equals

and

$$
Q(x)=C_{r-1}^{2}(x)
$$

$$
U_{n-1}(\cos \theta)=C_{n-1}^{1}(\cos \theta)=\frac{\sin (n \theta)}{\sin \theta} \text {. }
$$

So, avoiding the expression (1.1), we have to verify that $\left(\sigma_{n}[Q]\right)(t) \geqslant 0$ for all $t \geqslant 1, n=1,2, \ldots$ with $Q$ given by (2.15).

\section{GROUP THEORETIC PRELIMINARIES}

Let $U$ be a compact topological group with closed subgroup $K$ such that for each irreducible unitary representation $\pi$ of $U$ the representation space $\mathscr{H}(\pi)$ contains an at most one-dimensional subspace of $K$-fixed vectors. Then the pair $(U, K)$ is called a (compact) Gelfand pair. If $\mathscr{H}(\pi)$ contains a $K$-fixed vector $e$ of unit norm then the function

$$
\phi(u):=(\pi(u) e, e), \quad u \in U,
$$

is called the spherical function on $U$ with respect to $K$ associated with $\pi$. Let $d u$ denote Haar measure on $U$ such that $\int_{U} d u=1$ and let $L^{2}(U):=$ $L^{2}(U ; d u)$. By Schur's lemma, distinct spherical functions are mutually orthogonal elements of $L^{2}(U)$.

Call a function on $U U$-finite if it is a finite linear combination of functions $u \mapsto\left(\pi(u) e_{1}, e_{2}\right)$, where $\pi$ is an irreducible unitary representation of $U$ and $e_{1}, e_{2} \in \mathscr{H}(\pi)$. If $V$ is a closed subgroup of $U$ then the restriction of a $U$-finite function to $V$ is $V$-finite. The $U$-finite $K$-biinvariant functions $f$ on $U$ are precisely the finite linear combinations of spherical functions $\phi_{\delta}$ :

$$
f(u)=\sum_{\text {finite }}\left(\left\|\phi_{\delta}\right\|_{2}\right)^{-2} c_{\delta} \phi_{\delta}(u), \quad u \in U .
$$

Then

$$
c_{\delta}=\int_{U} f(u) \overline{\phi_{\delta}(u)} d u .
$$


A continuous function $f$ on $U$ is called positive definite on $U$ if

$$
\int_{U} \int_{U} f\left(u_{2}^{-1} u_{1}\right) d \mu\left(u_{1}\right) \overline{d \mu\left(u_{2}\right)} \geqslant 0
$$

for all complex Borel measures $\mu$ on $U$. Clearly, the restriction of a positive definite function on $U$ to a closed subgroup $V$ is positive definite on $V$. If the spherical function $\phi$ is given by $(3.1)$ and $\left\{e_{1}, \ldots, e_{n}\right\}$ is an orthonormal basis of $\mathscr{H}(\pi)$ then

$$
\phi\left(u_{2}^{-1} u_{1}\right)=\sum_{j=1}^{n}\left(\pi\left(u_{1}\right) e, e_{j}\right) \overline{\left(\pi\left(u_{2}\right) e, e_{j}\right)}, \quad u_{1}, u_{2} \in U .
$$

It follows that spherical functions are positive definite and also functions of the form (3.2) with all $c_{\delta} \geqslant 0$. Conversely, if $f$ is a positive definite function on $U$ and $\phi$ a spherical function then

$$
\begin{aligned}
\int_{U} f(u) \overline{\phi(u)} d u & =\int_{U} f\left(u_{2}^{-1} u_{1}\right) \overline{\phi\left(u_{2}^{-1} u_{1}\right)} d u_{1} \\
& =\int_{U} \int_{U} f\left(u_{2}^{-1} u_{1}\right) \overline{\phi\left(u_{2}^{-1} u_{1}\right)} d u_{1} d u_{2} \\
& =\sum_{j=1}^{n} \int_{U} \int_{U} f\left(u_{2}^{-1} u_{1} \overline{\left(\pi\left(u_{1}\right) e, e_{j}\right)}\left(\pi\left(u_{2}\right) e, e_{j}\right) d u_{1} d u_{2} \geqslant 0\right.
\end{aligned}
$$

for all $u_{2} \in U$. It follows in particular that $f$ of the form (3.2) is positive definite iff all $c_{\delta} \geqslant 0$.

We need a few facts about spherical functions on the sphere $S^{N-1}=$ $\mathrm{SO}(N) / \mathrm{SO}(N-1)$, which can be obtained from the theory of spherical harmonics, cf. for instance Müller [9]. Let $U:=\mathrm{SO}(N)$, the group of real orthogonal $N \times N$ matrices of determinant 1, with subgroups

and

$$
K:=\left(\begin{array}{cc}
1 & 0 \\
0 & \mathrm{SO}(N-1)
\end{array}\right)
$$

$$
A:=\left\{a_{\theta}=\left(\begin{array}{ccc}
\cos \theta & -\sin \theta & 0 \\
\sin \theta & \cos \theta & 0 \\
0 & 0 & I_{N-2}
\end{array}\right)\right\} .
$$

Suppose $N \geqslant 3$. Then $(U, K)$ is a Gelfand pair, there is the Cartan decomposition $U=K A K$, where $u \in U$ determines unique $\theta \in[0, \pi]$ such 
that $u \in K a_{\theta} K$, and, for $f \in C(U / / K)$ (continuous $K$-bi-invariant on $U$ ):

$$
\int_{U} f(u) d u=\frac{\Gamma\left(\frac{1}{2} N\right)}{\Gamma\left(\frac{1}{2}\right) \Gamma\left(\frac{1}{2} N-\frac{1}{2}\right)} \int_{0}^{\pi} f\left(a_{\theta}\right)(\sin \theta)^{N-2} d \theta .
$$

The spherical functions $\phi$ on $U$ with respect to $K$ are completely determined by their restrictions to $A$ and are there given by

$$
\phi\left(a_{\theta}\right)=C_{n}^{(1 / 2 N)-1}(\cos \theta) / C_{n}^{(1 / 2 N)-1}(1),
$$

where $n=0,1, \ldots$ and $C_{n}^{(1 / 2 N)-1}$ denotes a Gegenbauer polynomial.

\section{PROOF OF THE POSITIVITY RESULT}

In this section we make silent use of the group theoretic conventions and results summarized in Section 5. Let $U:=\mathrm{SO}(5)$ with subgroups

$$
\begin{gathered}
K:=\left(\begin{array}{cc}
1 & 0 \\
0 & \mathrm{SO}(4)
\end{array}\right), \quad M:=\left(\begin{array}{cc}
I_{2} & 0 \\
0 & \mathrm{SO}(3)
\end{array}\right), \\
A:=\left\{a_{\theta}=\left(\begin{array}{ccc}
\cos \theta & -\sin \theta & 0 \\
\sin \theta & \cos \theta & 0 \\
0 & 0 & I_{3}
\end{array}\right)\right\}, \\
B:=\left\{b_{\theta}=\left(\begin{array}{cccc}
1 & 0 & 0 & 0 \\
0 & \cos \theta & -\sin \theta & 0 \\
0 & \sin \theta & \cos \theta & 0 \\
0 & 0 & 0 & I_{2}
\end{array}\right)\right\},
\end{gathered}
$$

and let $\psi_{n}(n=1,2, \ldots)$ be the spherical functions on $K$ with respect to $M$ such that

$$
\psi_{n}\left(b_{\theta}\right)=C_{n-1}^{1}(\cos \theta) / C_{n-1}^{1}(1)=\frac{\sin (n \theta)}{n \sin \theta} .
$$

Let $p \in C(U / / K)$ and put

$$
P(\cos \theta):=p\left(a_{\theta}\right) .
$$

Then, for each $\eta$, the function $k \mapsto p\left(a_{\eta} k a_{\eta}^{-1}\right)$ is $M$-bi-invariant on $K$, 
since $A$ and $M$ commute. Hence, by (3.6), (4.1), (4.2):

$$
\begin{aligned}
\int_{K} p\left(a_{\eta} k a_{\eta}^{-1}\right) \psi_{n}(k) d k & =2 \pi^{-1} n^{-1} \int_{0}^{\pi} p\left(a_{\eta} b_{\theta} a_{\eta}^{-1}\right) \sin (n \theta) \sin \theta \mathrm{d} \theta \\
& =2 \pi^{-1} n^{-1} \int_{0}^{\pi} P\left(\cos ^{2} \eta+\sin ^{2} \eta \cos \theta\right) \sin (n \theta) \sin \theta d \theta .
\end{aligned}
$$

Compare with (2.4). Then it follows that

$$
\left(\sigma_{n}[P]\right)\left(\sin ^{-2} \eta\right)=n \sin ^{2} \eta \int_{K} p\left(a_{\eta} k a_{\eta}^{-1}\right) \psi_{n}(k) d k .
$$

For each $\eta,\left(a_{\eta} K a_{\eta}^{-1}, M\right)$ is a Gelfand pair with spherical functions $a_{\eta} k a_{\eta}^{-1} \mapsto \psi_{n}(k)$. Let $P$ be a polynomial (or, equivalently, $p$ a $U$-finite function in $C(U / / K))$. Then, by $(4.3),\left(\sigma_{n}[P]\right)(t) \geqslant 0$ for all $n, t$ iff $p$ restricted to any subgroup $a_{\eta} K a_{\eta}^{-1}$ is positive definite.

By Section 2, for settling the Milin conjecture, we have to verify that $\left(\sigma_{n}[Q]\right)(t) \geqslant 0$ for all $n, t$, with $Q$ given by (2.15). The corresponding $q \in C(U / / K)$ is given by

$$
q\left(k_{1} a_{\theta} k_{2}\right)=C_{r-1}^{2}(\cos \theta), \quad k_{1}, k_{2} \in K .
$$

We recognize the $C_{r-1}^{2}$ as a spherical function $\chi_{r}$ on $\mathrm{SO}(6)$ with respect to $\mathrm{SO}(5)$ (up to a positive factor). Let $G:=\mathrm{SO}(6), U$ embedded as a subgroup of $G$ by

and let

$$
U:=\left(\begin{array}{cc}
\mathrm{SO}(5) & 0 \\
0 & 1
\end{array}\right)
$$

$$
V:=\left(\begin{array}{cc}
1 & 0 \\
0 & \mathrm{SO}(5)
\end{array}\right) \text {. }
$$

Then $\chi_{r}\left(v_{1} a_{\theta} v_{2}\right)=C_{r-1}^{2}(\cos \theta), v_{1}, v_{2} \in V$. Since $V \cap U=K$, it follows that $\left.\chi_{r}\right|_{u}=q$, so $q$ restricted to $a_{\eta} K a_{\eta}^{-1}$, which equals $\chi_{r}$ restricted to $a_{\eta} K a_{\eta}^{-1}$, is positive definite for each $\eta$, since $\chi_{r}$ as a spherical function is positive definite. Hence $\left(\sigma_{n}[Q]\right)(t) \geqslant 0$ for all $n, t$ and our promises are fulfilled.

\section{THE SUM OF SQUARES}

In de Branges [4] and Koornwinder [8] it was pointed out that (1.3) multiplied by $s^{-n}$ also yields a solution of (2.1), while Askey and Gasper 
[1], [2] wrote (1.1) as a sum of expressions (1.3). These things also fit nicely into our picture of Section 4.

Replace $P, p$ in (4.2) and (4.3) by $Q, q$. In view of Proposition 2.2 and (4.3), any $U$-finite non-zero $q \in C(U / / K)$ which is positive definite on all subgroups $a_{\eta} K a_{\eta}^{-1}$ yields an admissible solution $\left\{\sigma_{n}\right\}$ of (2.1) and conversely. In particular, if $q$ is positive definite on $U$ then it is positive definite on all subgroups $a_{\eta} K a_{\eta}^{-1}$ (but not conversely), and the positive definite $U$-finite $q$ on $U$ are of the form

$$
q=\sum_{\text {finite }} a_{l} \phi_{l}, \quad a_{l} \geqslant 0,
$$

where $\phi_{l}$ is the spherical function on $U$ with respect to $K$ given by

$$
\phi_{l}\left(a_{\theta}\right):=C_{l-1}^{3 / 2}(\cos \theta) / C_{l-1}^{3 / 2}(1), \quad l=1,2, \ldots .
$$

Write $\Phi_{l}(\cos \theta):=\phi_{l}\left(a_{\theta}\right)$. Then, by (4.3):

$$
\left(\sigma_{n}\left[\Phi_{l}\right]\right)\left(\sin ^{-2} \eta\right)=n \sin ^{2} \eta \int_{K} \phi_{l}\left(a_{\eta} k a_{\eta}^{-1}\right) \psi_{n}(k) d k
$$

It follows from $[7, \S 3.3]$ that

$$
\int_{K} \phi_{l}\left(a_{\eta_{1}} k a_{\eta_{2}}^{-1}\right) \psi_{n}(k) d k=\phi_{l}^{n}\left(a_{\eta_{1}}\right) \overline{\phi_{l}^{n}\left(a_{\eta_{2}}\right)},
$$

where $\phi_{l}^{n}$ is an associated spherical function. Hence combination of (5.2) and (5.3) gives

$$
\left(\sigma_{n}\left[\Phi_{l}\right]\right)\left(\sin ^{-2} \eta\right)=n \sin ^{2} \eta\left|\phi_{l}^{n}\left(a_{\eta}\right)\right|^{2} .
$$

This shows once more that $\left(\sigma_{n}\left[\Phi_{l}\right]\right)(t) \geqslant 0$.

For $q$ of the form (5.1):

$$
\text { - }\left(\sigma_{n}[Q]\right)\left(\sin ^{-2} \eta\right)=n \sin ^{2} \eta \sum_{l} a_{l}\left|\phi_{l}^{n}\left(a_{\eta}\right)\right|^{2} .
$$

In particular, if $q$ is given by (4.4), then $q$, as the restriction of the positive definite spherical function $\chi_{r}$, is positive definite on $U$, so $a_{l} \geqslant 0$ in (5.1). Then (5.6) is the Askey-Gasper expansion of (1.1) in terms of (1.3).

The relationship between $\left|\phi_{l}^{n}\left(a_{\eta}\right)\right|^{2}$ and (1.3) can be seen more explicitly by rewriting (5.3) as

$$
\begin{aligned}
& \left(\sigma_{n}\left[\Phi_{l}\right]\right)\left(\sin ^{-2} \eta\right)=2 \pi^{-1}\left(C_{l-1}^{3 / 2}(1)\right)^{-1} \sin ^{2} \eta \\
& \times \int_{0}^{\pi} C_{l-1}^{3 / 2}\left(\cos ^{2} \eta+\sin ^{2} \eta \cos \theta\right) C_{n-1}^{1}(\cos \theta) \sin ^{2} \theta d \theta .
\end{aligned}
$$


We recognize the right-hand side of (5.7) as an integrated form of the addition formula for Gegenbauer polynomials [5,10.9 (34), watch for the misprint $2^{m}$ which should be $2^{2 m}$ and obtain

$$
\phi_{l}^{n}\left(a_{\eta}\right)=\operatorname{const}(\sin \eta)^{n-1} C_{l-n}^{n+(1 / 2)}(\cos \eta) .
$$

\section{A MORE GENERAL SYSTEM OF DIFFERENTIAL EQUATIONS AND ITS SOLUTIONS}

In [4] de Branges considers the system of differential equations

$$
\begin{aligned}
\sigma_{n}(t) & +n^{-1} t \sigma_{n}^{\prime}(t) \\
& =\frac{(2 v+n)(2 v+n+1)}{n(n+1)}\left(\sigma_{n+1}(t)-(2 v+n+1)^{-1} t \sigma_{n+1}^{\prime}(t)\right),
\end{aligned}
$$

$t \geqslant 1, n=1,2, \ldots, v>-\frac{1}{2}$. For $v=0$ it reduces to (2.1). Call a solution $\sigma_{n}$ admissible if $\sigma_{1}$ is not identically zero, $\sigma_{n}$ is identically zero for $n$ sufficiently large and $d / d t\left(t^{-2 v} \sigma_{n}(t)\right) \leqslant 0$ for all $t \geqslant 1, n=1,2, \ldots$ In $[4$, Theorem 2] de Branges states that any admissible solution of (6.1) yields an inequality for the coefficients $a_{n}$ in

$$
v^{-1}\left[(f(z))^{v}-\left(f^{\prime}(0) z\right)^{v}\right]=\sum_{n=1}^{\infty} a_{n} z^{v+n},
$$

where $f$ is a univalent analytic function on the unit disk sending 0 to 0 .

We can formulate a generalization of Theorem 2.1:

TheOrem 6.1 Let $P \in C^{1}([-1,1])$ and define functions $\sigma_{n}=\sigma_{n}[P]$ $(n=1,2, \ldots)$ on $[1, \infty)$ by

$$
\begin{aligned}
\sigma_{n}(t):= & \frac{n !(n-1) ! \Gamma(v+2)}{(2 v+1)_{n}(2 v+1)_{n-1} \pi^{1 / 2} \Gamma\left(v+\frac{3}{2}\right)} \\
& \times t^{-1} \int_{-1}^{1} P\left(1-t^{-1}(1-x)\right) C_{n-1}^{v+1}(x)\left(1-x^{2}\right)^{v+(1 / 2)} d x
\end{aligned}
$$

Then the functions $\sigma_{n}$ solve (6.1) with initial values

$$
\begin{aligned}
\sigma_{n}(1)= & \frac{n !(n-1) ! \Gamma(v+2)}{(2 v+1)_{n}(2 v+1)_{n-1} \pi^{1 / 2} \Gamma\left(v+\frac{3}{2}\right)} \\
& \times \int_{-1}^{1} P(x) C_{n-1}^{v+1}(x)\left(1-x^{2}\right)^{v+(1 / 2)} d x .
\end{aligned}
$$


Proof Let $\sigma_{n}$ be defined by (6.2). Then

$$
\begin{aligned}
& \sigma_{n}(t)+ n^{-1} t \sigma_{n}^{\prime}(t)-\frac{(2 v+n)(2 v+n+1)}{n(n+1)} \\
& \quad \times\left[\sigma_{n+1}(t)-(2 v+n+1)^{-1} t \sigma_{n+1}^{\prime}(t)\right] \\
&= \frac{n !(n-1) ! \Gamma(v+2) t^{-1}}{(2 v+1)_{n}(2 v+1)_{n-1} \pi^{1 / 2} \Gamma\left(v+\frac{3}{2}\right)} \int_{-1}^{1} P\left(1-t^{-1}(1-x)\right) \\
& \times\left[\left(1-n^{-1}(1-x) \frac{d}{d x}\right)\left(C_{n-1}^{v+1}(x)\left(1-x^{2}\right)^{v+(1 / 2)}\right)\right. \\
&\left.-\left(1+(2 v+n+1)^{-1}(1-x) \frac{d}{d x}\right)\left(C_{n}^{v+1}(x)\left(1-x^{2}\right)^{v+(1 / 2)}\right)\right] d x .
\end{aligned}
$$

The expression in brackets vanishes because of $[5,10.9$ (11), (35)].

Note that

$$
-t^{2 v+1} \frac{d}{d t}\left(t^{-2 v} \sigma_{n}[P]\right)(t)=\left(\sigma_{n}[Q]\right)(t)
$$

with

$$
Q(x)=-(1-x)^{-2 v} \frac{d}{d x}\left((1-x)^{2 v+1} P(x)\right) .
$$

We might now produce the various special $Q$ which yield the special admissible solutions considered in de Branges [4]. These $Q$ would again be spherical functions on spheres restricted to lower dimensional spheres, except that the dimension is now generally fractional, so that the group theoretic interpretation is only formal. We might still use grouplike arguments about positive definiteness, as in [6], but it is more straightforward to work with explicit "sum of squares" solutions generalizing (5.6). However, we will not pursue this line here.

\section{References}

[1] R. Askey and G. Gasper, Positive Jacobi polynomial sums, II, Amer. J. Math. 98 (1976), 709-737.

[2] R. Askey and G. Gasper, Inequalities for polynomials, in Proceedings of the Lafayette Meeting on Occasion of the Solution of the Bieberbach Conjecture (to appear).

[3] L. de Branges, A proof of the Bieberbach conjecture, Acta Math. 154 (1985), 137-152.

[4] L. de Branges, Powers of Riemann mapping functions, in Proceedings of the Lafayette Meeting on Occasion of the Solution of the Bieberbach Conjecture (to appear). 
[5] A. Erdélyi et al., Higher Transcendental Functions, Vol. II, McGraw-Hill, 1953.

[6] T. H. Koornwinder, Positivity proofs for linearization and connection coefficients of orthogonal polynomials satisfying an addition formula, J. London Math. Soc. (2) 18 (1978), 101-114.

[7] T. H. Koornwinder, Jacobi functions and analysis on noncompact semisimple Lie groups, in Special Functions: Group Theoretical Aspects and Applications, ed. by R. Askey et al., Reidel, Dordrecht, 1984, pp. 1-85.

[8] T. H. Koornwinder, Squares of Gegenbauer polynomials and Milin type inequalities, Report PM-R 8412, Centre for Math. and Computer Science, Amsterdam, 1984.

[9] C. Müller, Spherical Harmonics, Lecture Notes in Math. No. 17, Springer, 1966. 